\title{
Alpha Shape Analysis: Extracting Composition, Surface Area, and Volume Post Clustering
}

\author{
Evan Still and Peter Hosemann
}

University of California Berkeley, Berkeley, California, United States

Atom Probe Tomography (APT) has become a standard method for visualizing solute-rich microstructural features in a wide variety of materials due to its unique capability to probe 3 -dimensional spatial, chemical, and even isotopic information simultaneously at the nanoscale [1][2][3][4]. These properties relevant to material scientists are derived from mass/charge ratios and positions on the detector and are not directly measured as is the case for imaging techniques. APT is a technique that requires extensive computational efforts. Despite, APT's high degree of spatial resolution accurate quantification of nanoscale features is still a source of ongoing research with many groups developing new approaches and algorithms to improve the detection of atomic ordering and clustering [5][6]. While these new density-based clustering approaches have increased the capability of quantifying solute clusters through number density, size, and subcompositions, there still exists a discrepancy in the outputs when compared to concentration based methods, such as Isosurfaces which provides the aforementioned statistics as well as composition, volume, and surface area. Figure 1 presents a solution to this discrepancy in which a novel framework, AlphaShape Analysis (ASA), is applied post clustering which provides estimations of composition, volume, and surface area given the output of a conventional clustering algorithm.

For both concentration and density-based clustering approaches, a fundamental pre-processing step is the filtration of the relevant ions for clustering. In the case of isosurfaces, the filtration is performed with respect of the ion types used to generate a density distribution or the ions that are used for the concentration threshold. While in spatial search algorithms the filtering is performed externally and produces the spatial data that corresponds to only the ions of interest. In the latter, the filtration directly limits compositional analysis to the subcomposition of the ions selected. In the case of isosurfaces the generation of a mesh via the marching cubes algorithm enables recovery of the full composition as well as volume and surface area.

This constraint on compositional analysis following clustering has long been acknowledged as a dilemma, and algorithms such as the Envelopment method were designed specifically to account for such discrepancies [7]. The envelopment and erosion approach is a modification of maximum separation where two additional distance parameters are supplied. The first of which determines the inclusion of "nonclustered" ions of all species relative to the distance to the nearest clustered ion. However, as noted by the authors this results in an enlargement of the clusters, so they then erode the clusters by removing all ions within the erosion distance from the boundary [7][8]. This approach does not guarantee the original feature boundary is preserved, however the convex hull of the cluster has been identified by both Felfer et al's work on voronoi clustering, and Iman et al's density based clustering framework, as a potential solution [6][9]. It should be noted however, that the convex hull cannot account for concave features such as the internal cavity expected when identifying the shell of a core-shell structure or in identifying dislocations which possess a degree of curvature. Thus a generalization of the convex hull, the alpha shape, is utilized instead.

Conceived by Edelsbrunner et al, the alpha shape is generated according to a filtration of the delaunay triangulation for which the alpha parameter, $\alpha$, governs the filtration [10]. The filtration is as follows; for each simplex in the delaunay triangulation there is a known radius for the circumscribing sphere, $\sigma_{\mathrm{T}}$, and 
if $\sigma_{\mathrm{T}}<\alpha$ then that simplex is preserved, and the alpha shape is composed of all remaining simplices. This construction enables the removal of simplices which branch over low density regions and cavities in the original delaunay triangulation enabling concave meshes while allowing the convex hull to be reclaimed given an infinite value for alpha.

The production of the alpha shape for an individual cluster necessitates an additional input parameter, and thus for a set of $\mathrm{N}$ clusters an additional $\mathrm{N}$ parameters in total. To avoid manual selection of these additional parameters an automated heuristic is developed based on the volume of the alpha shape over a range of alphas. The heuristic is broken up into three requirements; 1 . All atoms in the original cluster are contained within the alpha shape, 2 . The Alpha shape is fully connected, and 3 . The Volume exists at a local minimum. In the event that there is not a sufficient alpha which satisfies these conditions, the cluster is identified as convex and the convex hull is returned. The application of this heuristic is demonstrated in Figure 2, where a sufficiently small results in violation of conditions 1 and 2 through the production of a highly fragmented volume, while an intermediate is identified which satisfies all three conditions.

In the ASA framework this process is repeated for each observed cluster and enables direct computation of the volume and surface area. Additionally, given the boundaries of the alpha shape and the ions initially omitted from clustering, rejection sampling can be employed to determine the composition on a cluster by cluster basis.

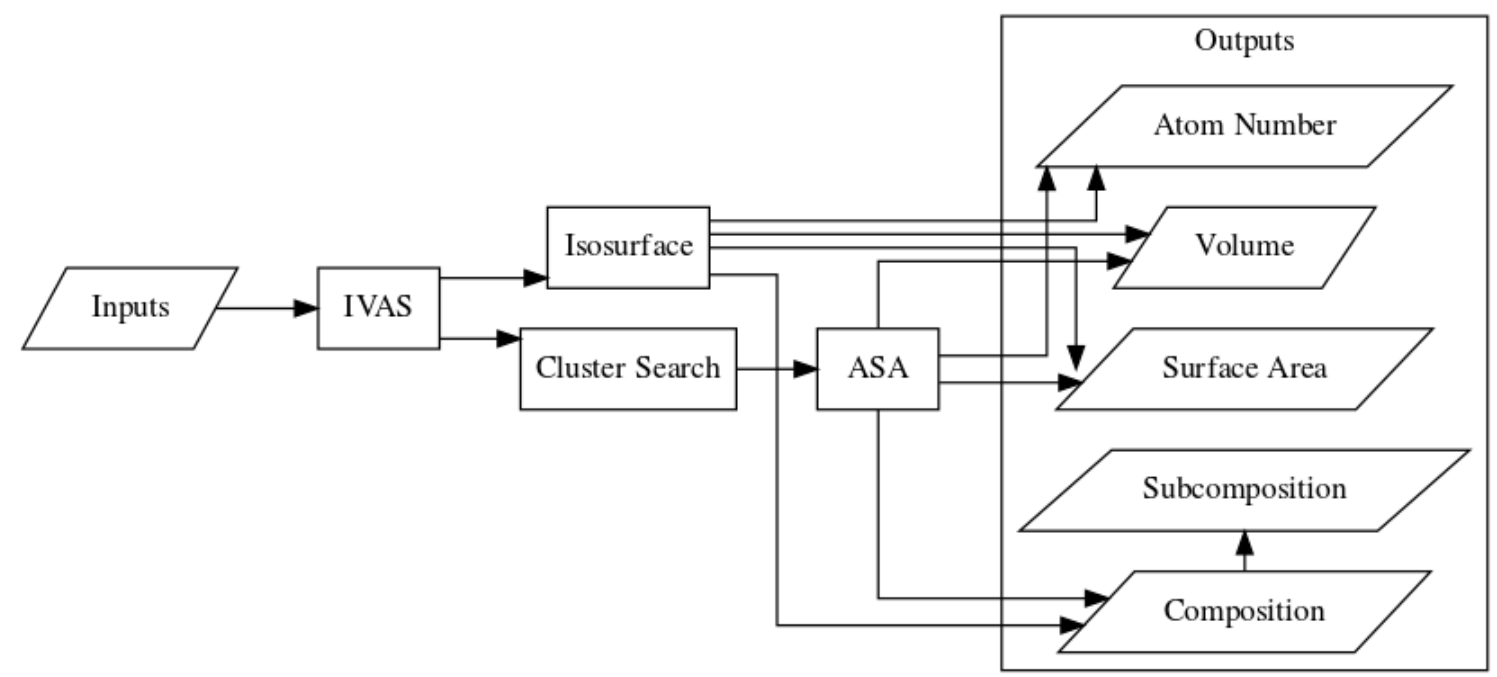

Figure 1. Role of Alpha Shape Analysis (ASA), in the analysis pipeline for APT data. Given the output of a cluster search, ASA provides additional information on the surface area, volume, and composition of clusters to allow similar property extraction to isosurfaces as implemented in IVAS. 


\section{Potential Alpha Shapes}
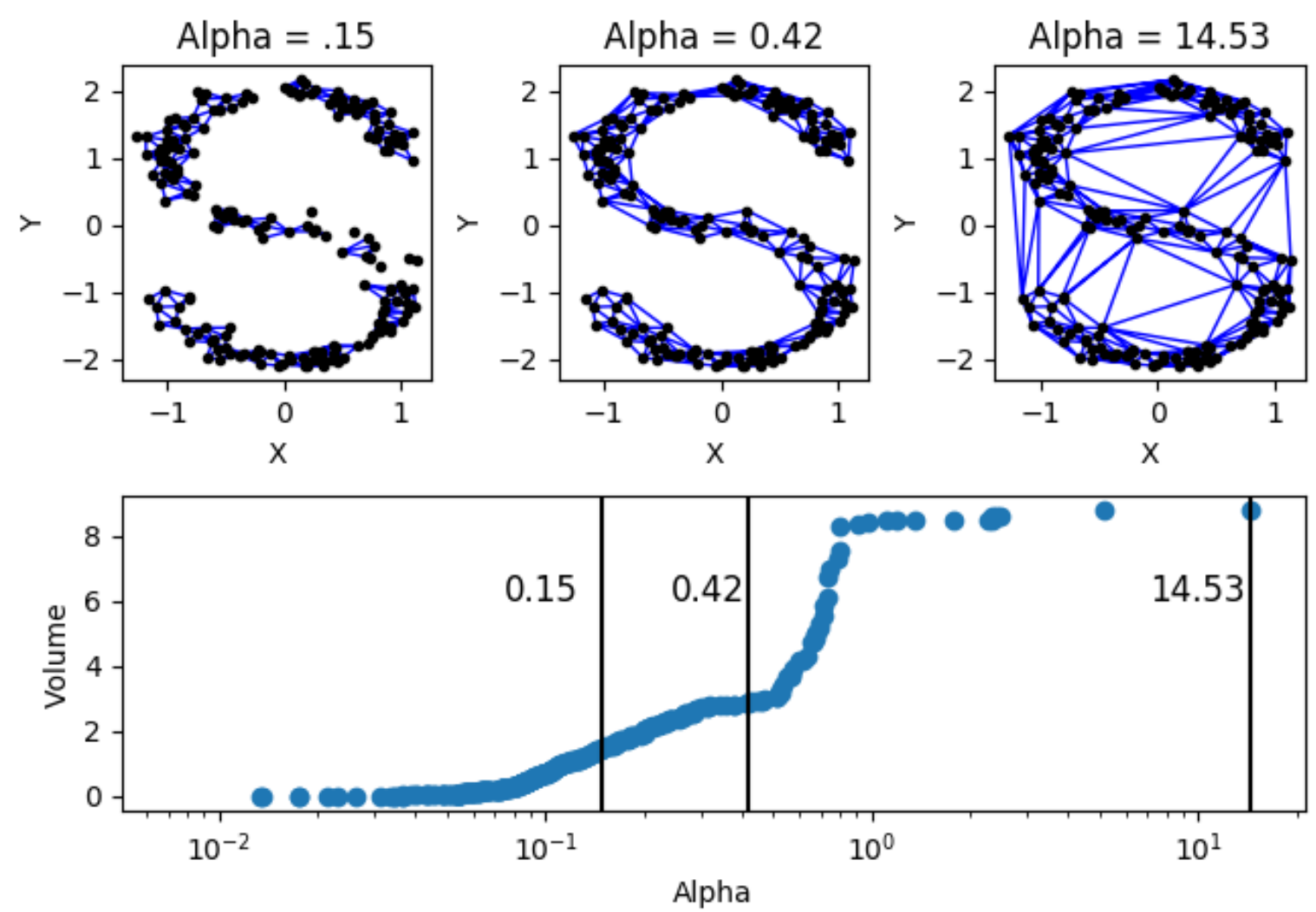

Figure 2. Example plot of the Complex Volume with respect to the selected alpha parameter for a 2-d scurve. The Alpha Complex for three example alphas are plotted to show a non-ideal fragmented complex $($ alpha $=.15)$, an ideal alpha as determined by our heuristic $($ alpha $=.42)$, and the convex complex (alpha $=14.53)$.

References

[1] B. P. Geiser et al., Microscopy and Microanalysis 13 (2007), p. 437 [2] B. P. Geiser B. P. et al., Microscopy and Microanalysis 15 (2009), p. 292 [3] E. A. Marquis and J. M. Hyde, Materials Science and Engineering: R: Reports 69 (2010), p. 37 [4] P. Bas et al., Applied Surface Science 87-88 (1995), p. 298 [5] J. Wang et al., Microscopy and Microanalysis 25 (2019), p. 338 [6] I. Ghamarian and E. A. Marquis, Ultramicroscopy 200 (2019), p. 28 [7] D. Vaumousse, A. Cerezo, and P. J. Warren, Ultramicroscopy 95 (2003), p. 215 [8] Z. Guo, W. Sha, and D. Vaumousse, Acta Materiala 51 (2003), p. 101 [9] P. Felfer et al., Ultramicroscopy 150 (2015), p. 30 [10] H. Edelsbrunner, D. Kirkpatrick, and R. Seidel, IEEE Transactions on Information Theory 29 (1983), p. 551 